\title{
Temperature effects in a nonlinear model of monolayer Scheibe aggregates
}

\author{
Bang, Ole; Christiansen, Peter Leth; If, F.; Rasmussen, Kim; Gaididei, Y. B.
}

Published in:

Physical Review E. Statistical, Nonlinear, and Soft Matter Physics

Link to article, DOI:

10.1103/PhysRevE.49.4627

Publication date:

1994

Document Version

Publisher's PDF, also known as Version of record

Link back to DTU Orbit

Citation (APA):

Bang, O., Christiansen, P. L., If, F., Rasmussen, K., \& Gaididei, Y. B. (1994). Temperature effects in a nonlinear model of monolayer Scheibe aggregates. Physical Review E. Statistical, Nonlinear, and Soft Matter Physics, 49(5), 4627-4636. https://doi.org/10.1103/PhysRevE.49.4627

\section{General rights}

Copyright and moral rights for the publications made accessible in the public portal are retained by the authors and/or other copyright owners and it is a condition of accessing publications that users recognise and abide by the legal requirements associated with these rights.

- Users may download and print one copy of any publication from the public portal for the purpose of private study or research.

- You may not further distribute the material or use it for any profit-making activity or commercial gain

- You may freely distribute the URL identifying the publication in the public portal 


\title{
Temperature effects in a nonlinear model of monolayer Scheibe aggregates
}

\author{
O. Bang, ${ }^{*}$ P. L. Christiansen, F. If, ${ }^{\dagger}$ and K. $\emptyset$. Rasmussen \\ Laboratory of Applied Mathematical Physics, Technical University of Denmark, DK-2800 Lyngby, Denmark \\ Y. B. Gaididei \\ Institute for Theoretical Physics, Metrologicheskaya Street 14 B, 252143 Kiev 143, Ukraine
}

(Received 19 October 1993; revised manuscript received 14 January 1994)

\begin{abstract}
A nonlinear dynamical model of molecular monolayers arranged in Scheibe aggregates is derived from a proper Hamiltonian. Thermal fluctuations of the phonons are included. The resulting equation for the excitons is the two dimensional nonlinear Schrödinger equation with noise. Two limits of the complicated spectrum of the noise are considered: time independent, spatially white noise, simply corresponding to disorder in the arrangement of the molecules, and pure white noise. Parameter values are found by comparison with experiments by Möbius and Kuhn [Isr. J. Chem. 18, 375 (1979)] and order of magnitude estimates given where experiments are not available. The temperature dependent coherence time is found from numerical simulations. Experiments show that the excitons stay coherent during their lifetime. This is in correspondence with the model at temperatures lower than $3 \mathrm{~K}$. To increase this limiting temperature it is found that the dipole-dipole coupling and the exciton-phonon coupling must be decreased significantly.
\end{abstract}

PACS number(s): 87.22.Bt, 05.40. $+\mathrm{j}, 63.20 . \mathrm{Kr}, 66.90 .+\mathrm{r}$

\section{INTRODUCTION}

Exciton motion in molecular systems is an important field of physics and has been undergoing active theoretical and experimental investigations. For a general review on excitons see, e.g., Davydov [1]. The field derives its importance from it being a part of the general area of energy transfer and its consequent connection with a variety of disciplines, even those outside physics, such as photosynthesis in biology [2].

The possibility of experimental studies of energy transfer in molecular systems was greatly influenced by the development of the Langmuir-Blodgett (LB) technique in the 1930s $[3,4]$. With this technique molecular thin films may be produced that consist of only a single layer of molecules. The molecules may be arranged in a predetermined structure and several layers may be stacked on each other. Thus LB films are particularly suited for studies of energy transfer, since the positions and orientations of the molecules are fixed and the distance between donors and acceptors can be varied systematically. For a general review on LB films and their many applications see, e.g., Tredgold [5] or Blinov [6].

In a particular kind of LB films, the molecules are arranged in so-called Scheibe aggregates [7], which is a special highly ordered and compact molecular structure,

\footnotetext{
*Present address: Laboratoire de Physique, Ecole Normale Superiéure de Lyon, 45 Allée d'Italie, 69364 Lyon Cédex 07, France.

†Present address: Cowi Consult, Parallelvej 15, DK-2800 Lyngby, Denmark.
}

reflecting itself in a strong narrow absorption band and almost coinciding fluorescence band of the film [8]. This characteristic Scheibe aggregate band (or $J$ band) will not be present in films where the molecular structure is less ordered and compact.

Molecular aggregates, such as Scheibe aggregates, are abundantly found in biological systems where they function as energy funnels for sunlight to be used in photochemical reactions [9]. Aggregates of dyes have also found technological application as sensitizers in the photographic industri [10] and recently they have shown promise for use in the field of photoelectronics [11].

Studying cyanine Scheibe aggregates, Möbius and Kuhn have observed highly efficient energy transfer over unusually large distances between donor and accepter molecules at room temperature $[12,13]$. The effect is up to ten times stronger when donors and acceptors are situated in the same layer than if situated in adjacent layers [13]. In both cases the efficiency of energy transfer is proportional to the temperature in the range $20-300 \mathrm{~K}$ [13]. In LB films with less ordered and compact molecular structure than in Scheibe aggregates, the energy transfer is less efficient, and no temperature dependence is observed in the range $20-300 \mathrm{~K}$ [12].

One of the main results of Möbius and Kuhn's work on cyanine Scheibe aggregates is that the excitons stay coherent during their lifetime and that the lifetime (radiative decay rate) of the exciton increases (decreases) with increasing temperature. They interpret this as a temperature dependent domain size of the exciton. This was also found by De Boer and Wiersma from studies of pseudoisocyanine bromide Scheibe aggregates [14]. Similar conclusions were drawn by Itoh, Ikehara, and Iwabuchi who studied the fluorescence lifetime of excitons in $\mathrm{CuCl}$ 
microcrystals [15], and by Feldman et al. [16], who studied the same effect in GaAlAs quantum wells. The experimental results reported in Refs. [14-16] were all obtained by spectroscopic measurements.

While in the past much work on Scheibe aggregates was aimed at understanding the spectroscopy [17], recently the focus has changed to comprehend the dynamical properties of these aggregates. Thus Mukamel and co-workers [18] have established the existence of a temperature dependent coherence size of the exciton from numerical calculations in one dimension, provided that the exciton dephasing time scale is much shorter than the fluorescence lifetime and that the exciton-phonon coupling is weak. When the coupling was strong, purely noncoherent motion is observed, with an effective coherence size of only one molecule, independent of temperature. The conclusion that the experimental results may only be reproduced if the exciton-phonon coupling is weak is also reached by Bartnik and Tuszynski, who studied a purely quantum mechanical model of Scheibe aggregates [19].

Huth, Gutmann, and Vitiello [20] have proposed a nonlinear model of Scheibe aggregates, where the coherent domain observed experimentally is supposed to arise from nonlinear dynamical effects in the aggregate. Thus the model is based on the cubic nonlinear Schrödinger (NLS) equation [21] with energy transfer occurring through solitary waves. The model was formulated in one dimension where the NLS equation is integrable and has soliton solutions [21]. However, it was supposed to hold also in two dimensions with the initial conditions becoming ring waves [22]. In two dimensions the NLS equation is no longer integrable, and thus the ring wave may collapse at the center [22]. Christiansen et al. [23] used this collapse of the ring waves in the nonlinear model to predict the lifetime of Möbius and Kuhn's exciton at room temperature. For a general review on collapse phenomena in NLS equations see, e.g., Rasmussen and Rypdal [24].

The nonlinear model of Huth, Gutmann, and Vitiello requires a strong coupling between excitons and phonons, which contrasts the result obtained by Mukamel, Bartnik, and their collaborators that the coupling must be weak. It is noted that the definition of weak and strong coupling is not clear in this connection. Furthermore, the model does not take thermal effects into account. Thus a natural question arises: Can solitary waves exist in Scheibe aggregates at room temperature?

It is the aim of the present work to answer this question and thereby clarify whether or not nonlinearity and strong exciton-phonon coupling may be consistent with coherent exciton motion in Scheibe aggregates. In particular the oxacyanine monolayer Scheibe aggregate studied experimentally by Möbius and Kuhn [13] will be used as physical example. The model is in terms of a postulated Hamiltonian, and thermal fluctuations of the phonons are taken into account. The resulting dynamical equation for the excitations becomes the two dimensional NLS equation with noise, where the noise has a rather complex spectrum. The initial excitation of the aggregate is modeled by the ground state solitary wave (GS) solution.
The time evolution of the excitation, determined by numerical solution of the two dimensional (2D) NLS equation, is compared with the experimentally observed domain of coherence. Parameters in the model are determined from experimental results or by orders of magnitude estimates. In particular the temperature dependence of the coherence time is investigated.

\section{DERIVATION OF THE MODEL}

The derivation follows closely that of the Davydov model of energy transport in protein [25], except that the present system is two dimensional and that thermal fluctuations of the molecules are taken into account. Thus the following energy operator is considered

$$
\hat{H}=\hat{H}_{\mathrm{ex}}+\hat{H}_{\mathrm{ph}}+\hat{H}_{\mathrm{int}} \text {. }
$$

The first component of $\hat{H}$ is the exciton energy operator

$$
\hat{H}_{\mathrm{ex}}=\sum_{n} E_{0} \hat{B}_{n}^{\dagger} \hat{\boldsymbol{B}}_{n}-\sum_{n \neq p} \sum_{p} J_{n p} \hat{\boldsymbol{B}}_{n}^{\dagger} \widehat{B}_{p},
$$

where $n$ and $p$ count molecules on the aggregate $(n, p=1,2, \ldots, f)$ and $f$ is the total number of molecules considered. $\hat{\boldsymbol{B}}_{n}^{\dagger}\left(\hat{\boldsymbol{B}}_{n}\right)$ are creation (annihilation) operators, $E_{0}$ is the molecular site energy, and $-J_{n p}$ is the dipole-dipole interaction energy. The phonon energy, which is treated classically, is given by

$$
\hat{H}_{\mathrm{ph}}=\frac{1}{2} M \sum_{n}\left[\dot{u}_{n}^{2}+\omega_{0}^{2} u_{n}^{2}\right],
$$

where $M$ is the molecular mass and $u_{n}(t)$ represents the elastic degree of freedom at site $n$. The molecules are approximated by Einstein oscillators, all oscillating at the same frequency $\omega_{0}$ and thus only optical phonons are taken into account [26]. Finally the exciton-phonon interaction energy operator is

$$
\hat{H}_{\text {int }}=\chi \sum_{n} u_{n} \hat{B}_{n}^{\dagger} \hat{B}_{n},
$$

where $\chi$ is the exciton-phonon coupling parameter.

The classical Hamiltonian is given by

$$
H=\operatorname{Tr}\{\widehat{\rho}(t) \hat{H}\} \text {, }
$$

where $\hat{\rho}(t)$ is a nonequilibrium density matrix [27] and $\operatorname{Tr}$ denotes the trace. Introducing the classical function

$$
\rho_{n n^{\prime}}(t)=\operatorname{Tr}\left\{\hat{\rho}(t) \widehat{B}_{n}^{\dagger} \widehat{B}_{n^{\prime}}\right\},
$$

Hamilton's equation for $H$, given by Eq. (5), gives the equation for $u_{n}$

$$
M \ddot{u}_{n}+M \omega_{0}^{2} u_{n}=\chi \rho_{n n} .
$$

To describe the interaction of the phonon system with a thermal reservoir at temperature $T$, damping $\lambda$ and noise $\eta_{n}(t)$ are included

$$
M \ddot{u}_{n}+M \lambda \dot{u}_{n}+M \omega_{0}^{2} u_{n}=\chi \rho_{n n}+\eta_{n} .
$$

Note that the noise is additive, implying that phonons are being created and destroyed. $\eta_{n}(t)$ is assumed to be 
Gaussian white noise with zero mean and the autocorrelation function

$$
\left\langle\eta_{n}(t) \eta_{n^{\prime}}\left(t^{\prime}\right)\right\rangle=2 M \lambda k T \delta\left(t-t^{\prime}\right) \delta_{n n^{\prime}},
$$

where the strength is chosen according to the classical fluctuation-dissipation theorem [28], which ensures thermal equilibrium. The damping coefficient $\lambda$ is the linewidth of the infrared absorption peak and $k$ is Boltzmann's constant.

Inserting the Hamiltonian, given by Eq. (1), in the quantum mechanical Liouville equation for the nonequilibrium density matrix

$$
i \hbar \frac{d \hat{\rho}}{d t}=[\hat{H}, \hat{\rho}],
$$

and equation for $\rho_{n n^{\prime}}(t)$ is obtained

$$
\begin{gathered}
i \hbar \dot{\rho}_{n n^{\prime}}=\sum_{p \neq n} J_{p n} \rho_{p n^{\prime}}-\sum_{p \neq n^{\prime}} J_{p n^{\prime}} \rho_{p n} \\
+\chi u_{n} \rho_{n n^{\prime}}-\chi u_{n^{\prime}} \rho_{n n^{\prime}}
\end{gathered}
$$

Assuming that $\rho_{n n^{\prime}}(t)$ may be written as a product

$$
\rho_{n n^{\prime}}(t)=\phi_{n}^{*}(t) \phi_{n^{\prime}}(t),
$$

Eqs. (8) and (11) lead to two coupled equations for the excitons and phonons

$$
\begin{aligned}
& i \hbar \dot{\phi}_{n}+\sum_{p \neq n} J_{p n} \phi_{p}+\chi u_{n} \phi_{n}=0, \\
& M \ddot{u}_{n}+M \lambda \dot{u}_{n}+M \omega_{0}^{2} u_{n}=\chi\left|\phi_{n}\right|^{2}+\eta_{n},
\end{aligned}
$$

where $\left|\phi_{n}(t)\right|^{2}$ is the probability for finding the exciton at site number $n$. Equations (13) and (14) resembles the one dimensional Davydov equations solved numerically by Lomdahl and Kerr [29]. Their conclusion was that Davydov solitons do not exist for sufficiently long time at $300 \mathrm{~K}$ to be of physical interest. In this case we derive a single equation describing the dynamics of the exciton system.

The solution to Eq. (14) is easily written down

$$
\begin{gathered}
u_{n}(t)=c_{1} e^{-\lambda t / 2} \cos \left(\omega_{1} t\right)+c_{2} e^{-\lambda t / 2} \sin \left(\omega_{1} t\right) \\
+\frac{1}{M \omega_{1}} \int_{0}^{t}\left[\chi\left|\phi_{n}(\tau)\right|^{2}+\eta_{n}(\tau)\right] e^{-\lambda(t-\tau) / 2} \\
\times \sin \left(\omega_{1}[t-\tau]\right) d \tau,
\end{gathered}
$$

where $c_{1}$ and $c_{2}$ are constants of the initial state, and the perturbed eigenfrequency $\omega_{1}$ is given by

$$
\omega_{1}=\omega_{0} \sqrt{1-\epsilon^{2}}, \epsilon=\lambda /\left(2 \omega_{0}\right) .
$$

The parameter $\epsilon$ is normally small for solids.

Assuming that $\left|\phi_{n}(t)\right|^{2}$ varies slowly compared with $\sin \left(\omega_{1} t\right)$, the solution to Eq. (15) becomes

$$
\begin{aligned}
u_{n}(t)= & \widehat{c}_{1} e^{-\lambda t / 2} \cos \left(\omega_{1} t\right)+\widehat{c}_{2} e^{-\lambda t / 2} \sin \left(\omega_{1} t\right) \\
& +\frac{\chi}{M \omega_{0}^{2}}\left|\phi_{n}(t)\right|^{2}+s_{n}(t),
\end{aligned}
$$

where the redefined coefficients $\widehat{c}_{1}$, and $\widehat{c}_{2}$ are given by

$$
\begin{aligned}
& \widehat{c}_{1}=c_{1}-\frac{\chi}{M \omega_{0}^{2}}\left|\phi_{n}(0)\right|^{2}, \\
& \hat{c}_{2}=c_{2}-\frac{\chi}{M \omega_{0}^{2}} \frac{\epsilon}{\sqrt{1-\epsilon^{2}}}\left|\phi_{n}(0)\right|^{2} .
\end{aligned}
$$

The noise $s_{n}(t)$ is not white, but strongly colored. It can be found from Eqs. (9) and (17), and is most conveniently expressed in Fourier space

$$
\widetilde{s}_{n}(\omega)=\frac{1}{M} \widetilde{f}(\omega) \widetilde{\eta}(\omega),
$$

where $\widetilde{f}(\omega)$ is the Lorentzian

$$
\widetilde{f}(\omega)=\frac{1}{\omega_{0}^{2}-\omega^{2}+i \lambda \omega}
$$

and the tilde denotes the Fourier transformation.

The first two terms on the right-hand side of Eq. (17) are the solution of the homogeneous part of Eq. (14). They are fast oscillating, with the perturbed eigenfrequency $\omega_{1} \leq \omega_{0}$, while the third term, proportional to $\left|\phi_{n}(t)\right|^{2}$, varies slowly. To remove the fast oscillating part, we average over a period of the oscillations and obtain the solution

$$
\begin{aligned}
& \bar{u}_{n} \equiv \frac{1}{t_{1}} \int_{t}^{t+t_{1}} u_{n}(\tau) d \tau=\frac{\chi}{M \omega_{0}^{2}}\left|\phi_{n}(t)\right|^{2}+\bar{s}_{n}(t), \\
& t_{1}=\frac{2 \pi}{\omega_{1}}
\end{aligned}
$$

Thus the modulus of the exciton wave function is the slowly varying mean value of the rapidly fluctuating phonons. The averaged noise is still exact.

Inserting the solution, given by Eq. (21), into the equation for the exciton wave function, Eq. (13), the discrete self-trapping equation (DST) [30] is obtained

$$
i \hbar \dot{\phi}_{n}+\sum_{p \neq n} J_{p n} \phi_{p}+V\left|\phi_{n}\right|^{2} \phi_{n}=-\sigma_{n} \phi_{n},
$$

where the nonlinearity parameter $V$ is given by

$$
V=\frac{\chi^{2}}{M \omega_{0}^{2}} .
$$

Without the assumption that $e^{-\lambda t / 2}$ varies slowly compared with $\cos \left(\omega_{1} t\right), V$ would become time dependent. The noise $\sigma_{n}(t)$ is again given in Fourier space

$$
\widetilde{\sigma}_{n}(\omega)=\frac{\chi}{M} \frac{\sin \left(\pi \omega / \omega_{1}\right)}{\pi \omega / \omega_{1}} e^{i \pi \omega / \omega_{1}} \widetilde{f}(\omega) \widetilde{\eta}_{n}(\omega) .
$$

Note that averaging over a period $2 \pi / \omega_{1}$ in real space leads to multiplication by $\sin \left(\pi \omega / \omega_{1}\right) /\left(\pi \omega / \omega_{1}\right)$ in Fourier space, with zeros at the harmonics of $\omega_{1}$. This is seen in Fig. 1(a), where the solid curve is the power spectrum of the noise before averaging $s_{n}(t)$ and the dashed curve is the influence of averaging. The power spectrum of the resulting noise $\sigma_{n}(t)$ is shown in Fig. 1(b).

In Eq. (22) the exciton number $N$, defined as the total probability for finding the exciton in the system 

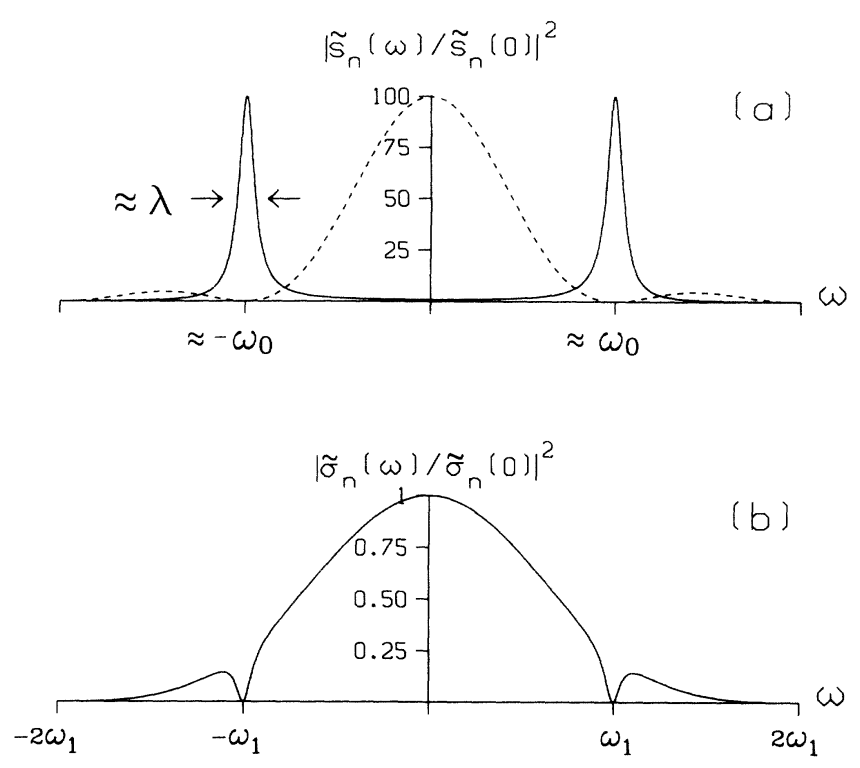

FIG. 1. (a) Power spectrum of the noise before averaging, $\left|\widetilde{s}_{n}(\omega)\right|^{2} /\left|\widetilde{s}_{n}(0)\right|^{2} \quad$ (solid), and $100 \quad\left[\sin \left(\pi \omega / \omega_{1}\right) /\left(\pi \omega / \omega_{1}\right)\right]^{2}$ (dashed). (b) Power spectrum of the resulting noise, $\left|\widetilde{\sigma}_{n}(\omega)\right|^{2} \omega_{0}=10^{12} \mathrm{~s}^{-1}$, and $\lambda=10^{11} \mathrm{~s}^{-1}$.

$$
N \equiv \sum_{n}\left|\phi_{n}(t)\right|^{2}=1
$$

is a conserved quantity, reflecting that the noise is multiplicative and only disturbs the coherence of the wave function.

Taking only nearest neighbor coupling $J_{0}$ into account and making the gauge transformation $\phi_{n} \rightarrow e^{i 4 J_{0} t / \hbar} \phi_{n}$, Eq. (22) may be approximated by the 2D NLS equation

$$
i \hbar \phi_{t}+l^{2} J_{0} \nabla^{2} \phi+l^{2} V|\phi|^{2} \phi=-l^{2} \sigma \phi,
$$

where $|\phi(x, y, t)|^{2}=\left|\phi_{n}(t)\right|^{2} / l^{2}$ is a probability density

$$
N=\iint|\phi(x, y, t)|^{2} d x d y=1
$$

and $\sigma(x, y, t)=\sigma_{n}(t) / l^{2}$ a noise density. $l$ is the distance between nearest neighboring molecules in the Scheibe aggregate.

\section{INITIAL CONDITION}

The initial excitation of the Scheibe aggregate is modeled by the GS solution to the 2D NLS equation. The GS solution is chosen because it represents a localized domain of coherence, as is also found experimentally, and it has the smallest value of $N$ of all solitary wave solutions. This solution will then propagate over the aggregate, its dynamics being determined by the 2D NLS equation as long as it stays coherent.

The 2D NLS equation is not integrable and possess unstable solutions that may collapse in finite time. Correspondingly the GS solution has not been found analytically. However, using a variational approach [31], Anderson, Bonnedal, and Lisak have derived the approximative form of the GS solution [32]

$$
\phi_{s}(r, t)=\kappa A_{0} \operatorname{sech}\left[\kappa \frac{r}{B_{0}}\right] e^{i \kappa^{2} J_{0} t / \hbar}
$$

here given with zero initial velocity and center at $r=0$. The initial amplitude $A_{0}$ and the initial width $B_{0}$ are given by

$$
\begin{aligned}
& A_{0}=\frac{1}{l}\left(\frac{J_{0}}{V} \frac{12 \ln (2)}{4 \ln (2)-1}\right]^{1 / 2}, \\
& B_{0}=l\left(\frac{2 \ln (2)+1}{6 \ln (2)}\right)^{1 / 2}
\end{aligned}
$$

The real parameter $\kappa$ reflects a similarity transformation, under which the NLS equation without noise is unchanged [33]. For $\phi_{s}$ given by Eq. (28) it follows that

$$
N=\iint\left|\phi_{s}\right|^{2} d x d y=11.73 \frac{J_{0}}{V} .
$$

Thus in order to fulfill Eq. (27) we must have

$$
\frac{V}{J_{0}}=11.73 \text {. }
$$

The exact value of $N$ for the GS solution has been found numerically to $N \equiv N_{s}=11.69 J_{0} / V$ [34]. For real initial conditions with $N$ larger (smaller) than $N_{s}$ the solution collapses in finite time (disperses) [24]. Since $N>N_{s}$ for $\phi_{s}$ the solution will eventually collapse, though the collapse time will be large. Even if the exact solution could be found, a small perturbation, such as that introduced by discretization in numerical simulations, would cause a localization in finite time. This interplay between discretization and collapse is studied in [35]. However, for all numerical simulations presented here, the collapse time of $\phi_{s}$ is much larger than the considered coherence time. Thus $\phi_{s}$ represents a time independent solitary wave solution.

\section{MODEL PARAMETERS}

In this section the values of the parameters appearing in the 2D NLS equation, Eq. (26), are estimated for the monolayer oxacyanine Scheibe aggregate studied by Möbius and Kuhn [13]. The aggregate is represented as a brickstone work [7], each brick being a molecule, and the molecules are represented as extended dipoles [36], as shown in Fig. 2. Experimental data are listed in Table I.
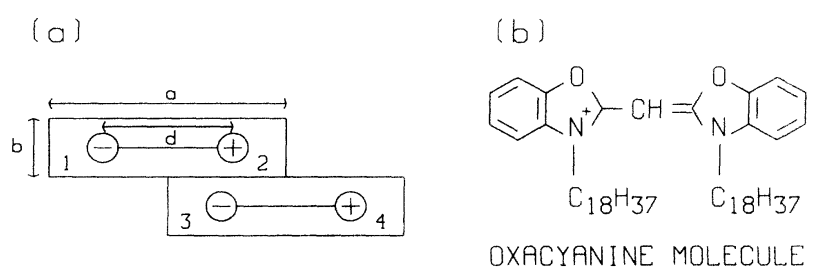

FIG. 2. Left: brickstone work representation of a monolayer Scheibe aggregate, with each molecule represented as an extended dipole. Right: oxacyanine molecule. 
TABLE I. Physical parameters for the oxacyanine Scheibe aggregate used in Möbius and Kuhn's experiment [13].

\begin{tabular}{llc}
\hline \hline Brickstone length & $a$ & $15.5 \AA$ \\
Brickstone width & $b$ & $4.0 \AA$ \\
Dipole length & $d$ & $8.9 \AA$ \\
Transition dipole moment & $\mu_{\mathrm{tr}}$ & $3.13 \times 10^{-29} \mathrm{C} \mathrm{m}$ \\
Dielectric constant of the aggregate & $\epsilon_{r}$ & 2.25 \\
\hline \hline
\end{tabular}

From Fig. 2 and Table I, the distance between nearest neighboring molecules $l$ is found to be

$$
l=\sqrt{a^{2} / 4+b^{2}}=8.72 \AA
$$

and the mass of an oxacyanine molecule is

$M=49[\mathrm{C}]+83[\mathrm{H}]+2[\mathrm{~N}]+2[\mathrm{O}]=1.21 \times 10^{-24} \mathrm{~kg}$,

where [ ] denotes the atomic mass.

Since $l$ is comparable with the size of the molecules, a point dipole approximation would be inadequate. Instead the molecules are approximated by extended dipoles [7], with the equivalent dipole charge $\mu_{\mathrm{tr}} / d$, where $\mu_{\mathrm{tr}}$ is the transition dipole moment and $d$ the equivalent dipole length. This gives interaction energies in good agreement with the results from quantum mechanical computations, by which Czikkely, Försterling, and Kuhn found that $\mu_{\mathrm{tr}}=3.13 \times 10^{-29} \mathrm{Cm}$ and $d=8.9 \AA$ for oxacyanine molecules $[36,13]$. The dipole-dipole interaction energy $J_{0}$ can thus be found from static calculations

$$
\begin{aligned}
J_{0} & =-\frac{\left(\mu_{\mathrm{tr}} / d\right)^{2}}{4 \pi \epsilon_{r} \epsilon_{0}}\left[\frac{1}{r_{13}}+\frac{1}{r_{24}}-\frac{1}{r_{23}}-\frac{1}{r_{14}}\right] \\
& =3.6 \times 10^{-21} \mathrm{~J} .
\end{aligned}
$$

The phonon eigenfrequency $\omega_{0}$ and the linewidth of the infrared absorption spectrum $\lambda$ are not known. However, in solids common values are of the order of 100 and 10 $\mathrm{cm}^{-1}$, respectively. Thus $\omega_{0}=10^{12} \mathrm{~s}^{-1}$ and $\lambda=10^{11} \mathrm{~s}^{-1}$ will be used as order of magnitude estimates.

The Davydov model of energy transport in protein by solitons [25] has been studied over two decades and complete agreement has not yet been reached on the value of the exciton-phonon coupling parameter $\chi$. The best currently available value is in the range $35-62 \mathrm{pN}$ [37]. The application of nonlinear dynamical models in the study of Scheibe aggregates is fairly new and correspond- ingly no estimates of $\chi$ have been published yet. Thus squinting at the Davydov model, the value $10^{-10} \mathrm{~N}$ will be used as an order of magnitude estimate of $\chi$. The nonlinearity parameter $V$ is found from Eq. (23).

The parameter values, summarized in Table II, are assumed to be temperature independent. Only the noise strength $\sigma(x, y, t)$ will be temperature dependent in our model

From the values given in Table II it follows that

$$
\frac{V}{J_{0}}=2.3 \text {, }
$$

which is a factor of 5 smaller than the value given in Eq. (31). In view of the fact that $V \propto \chi^{2}$, and given by an order of magnitude estimate, a factor of 5 is acceptable.

\section{APPROXIMATIONS OF THE NOISE}

Usually, when noise is simulated numerically, it is defined in Fourier space and then transformed back to real space [38]. In two dimensions, and for the large number of molecules we want to consider, this would require an excessive amount of storage capacity. However, in the two limiting cases of time independent and pure white noise, this problem is easily overcome. The time independent noise is only calculated initially and the white noise can be generated for each time step by a random noise generator. In Fig. $3\left|\widetilde{\sigma}_{n}(\omega)\right|^{2}$ is shown in the two cases.

We first approximate the power spectrum of the noise in Eq. (22), $\sigma_{n}(t)$, with a $\delta$ function with the strength equal to the integral over the interval $-\omega_{1} \leq \omega \leq \omega_{1}$,

$\left|\widetilde{\sigma}_{n}(\omega)\right|^{2} \approx \frac{4 \omega_{1}}{3}\left|\widetilde{\sigma}_{n}(0)\right|^{2} \delta(\omega)=\frac{8 \omega_{1} \chi^{2} \lambda k T}{3 M \omega_{0}^{4}} \delta(\omega)$,

where $\left|\widetilde{\sigma}_{n}(0)\right|$ is obtained from Eq. (24). This simply correspond to disorder in the arrangement of the molecules

TABLE II. Estimated values of the parameters in the nonlinear model of the monolayer oxacyanine Scheibe aggregate used in Möbius and Kuhn's experiment [13]. An asterisk indicates that the value is an order of magnitude estimate.

\begin{tabular}{llc}
\hline \hline Molecular mass & $M$ & $1.2 \times 10^{-24} \mathrm{~kg}$ \\
Distance between molecules & $l$ & $8.72 \AA$ \\
Phonon eigenfrequency $^{*}$ & $\omega_{0}$ & $10^{12} \mathrm{~s}^{-1}$ \\
Linewidth of infrared absorption spectrum ${ }^{*}$ & $\lambda$ & $10^{11} \mathrm{~s}^{-1}$ \\
Exciton-phonon coupling parameter & $\chi$ & $10^{-10} \mathrm{~N}$ \\
Dipole-dipole coupling energy & $\chi$ & $3.6 \times 10^{-21} \mathrm{~J}$ \\
Nonlinearity parameter & $J_{0}$ & $8.3 \times 10^{-21} \mathrm{~J}$ \\
\hline \hline
\end{tabular}




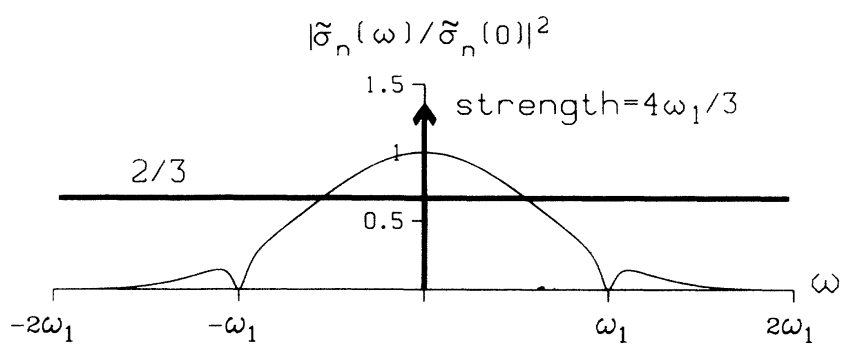

FIG. 3. Power spectrum of the noise, $\left|\widetilde{\sigma}_{n}(\omega)\right|^{2} /\left|\widetilde{\sigma}_{n}(0)\right|^{2}$. White noise $\left[\left|\widetilde{\sigma}_{n}(\omega)\right|^{2}=\frac{2}{3}\right]$ and time independent noise $\left[\left|\widetilde{\sigma}_{n}(\omega)\right|^{2}=\left(4 \omega_{1} / 3\right) \delta(\omega)\right]$ approximations are indicated. Parameters are taken from Table II.

in the aggregate. Transforming back and making the continuum approximation leads to Eq. (26), in which the noise density $\sigma(x, y, t)$ has the autocorrelation function

$$
\left\langle\sigma(x, y, t) \sigma\left(x^{\prime}, y^{\prime}, t^{\prime}\right)\right\rangle=D_{\text {disorder }} \delta\left(x-x^{\prime}\right) \delta\left(y-y^{\prime}\right) .
$$

Here the temperature dependent variance $D_{\text {disorder }}$ is given by

$$
D_{\text {disorder }}=\frac{4 \omega_{1} \chi^{2} \lambda k T}{3 \pi M \omega_{0}^{4} l^{2}} .
$$

At $T=300 \mathrm{~K}$ the strength of the noise is $\sqrt{l^{2} D_{\text {disorder }}}=1.2 \times 10^{-21} \mathrm{~J}$, which is of the same order of magnitude as $J_{0}$ and $V$. With this approximation of the noise an analytical estimate of the coherence time cannot be obtained.

In the pure white noise approximation the power spectrum of the noise in Eq. $(22), \sigma_{n}(t)$, is constant and given by the mean value in the interval $-\omega_{1} \leq \omega \leq \omega_{1}$,

$$
\left|\widetilde{\sigma}_{n}(\omega)\right|^{2} \approx \frac{2}{3}\left|\widetilde{\sigma}_{n}(0)\right|^{2}=\frac{4 \chi^{2} \lambda k T}{3 M \omega_{0}^{4}} .
$$

In this case the autocorrelation function for the noise density in Eq. (26) becomes

$$
\begin{aligned}
& \left\langle\sigma(x, y, t) \sigma\left(x^{\prime}, y^{\prime}, t^{\prime}\right)\right\rangle \\
& \quad=D_{\text {white }} \delta\left(x-x^{\prime}\right) \delta\left(y-y^{\prime}\right) \delta\left(t-t^{\prime}\right) .
\end{aligned}
$$

Here the temperature dependent variance $D_{\text {white }}$ is given by

$$
D_{\text {white }}=\frac{4 \chi^{2} \lambda k T}{3 M \omega_{0}^{4} l^{2}} .
$$

Introducing a deterministic function $\psi(x, y, t)$ describing the coherence of the stochastic wave function $\phi(x, y, t)$ through

$$
\left\langle\phi^{*}(x, y, t) \phi\left(x^{\prime}, y^{\prime}, t\right)\right\rangle=\psi^{*}(x, y, t) \psi\left(x^{\prime}, y^{\prime}, t\right),
$$

we obtain [39-41]

$$
i \hbar \psi_{t}+l^{2} J_{0} \nabla^{2} \psi+l^{2} V|\psi|^{2} \psi=-i l^{2} \hbar \Gamma \psi,
$$

when the noise $\sigma(x, y, t)$ in Eq. (26) is approximated with white noise for

$$
J_{0} \gg D_{\text {white }}
$$

The loss parameter $\Gamma$ is connected to the variance $D_{\text {white }}$ through the simple relation

$$
\Gamma=\frac{1}{2 \hbar^{2}} D_{\text {white }}
$$

In Eq. (43) $N$ decays exponentially $N=N_{0} e^{-2 \Gamma t}$, indicating that the coherence of the exciton is being destroyed by collisions with the fluctuating phonons. This allows us to estimate the coherence time as

$$
t_{\mathrm{coh}}=\frac{1}{2 l^{2} \Gamma}=\frac{\hbar^{2}}{l^{2} D_{\text {white }}}=2.4 \times 10^{-15} \mathrm{~s}\left(\frac{300 \mathrm{~K}}{T}\right) .
$$

Clearly the white noise approximation is poorer than the disorder approximation since it has infinite energy, in contrast to the finite energy of the real noise. Thus it is expected that the white noise will lead to an extremely short coherence time of the exciton, which is also predicted by Eq. (45) $(2.4$ fs at $T=300 \mathrm{~K})$. However, it represents a limiting case (shortest coherence time).

\section{NUMERICAL RESULTS}

The 2D NLS equation, Eq. (26), is solved numerically with a second order split-step Fourier scheme [42,41] and using the time independent and white noise approximations. The approximation of the GS solution, Eqs. (28) and (29), is used as initial condition.

Figure 4 depicts the time evolution of the distribution of the probability amplitude $|\phi(x, y, t)|^{2}$ for the time independent noise (right) and white noise (left) at room temperature. The difference between the energy content of the noise in the two approximations is clearly seen. For white noise the exciton is scattered very rapidly and coherence is completely lost after approximately $10^{-14} \mathrm{~s}$. However, the solution stays coherent considerably longer than predicted analytically $\left(2.4 \times 10^{-15} \mathrm{~s}\right)$, as also found in $[40,41]$. For the time independent noise the solution remains coherent for approximately $10^{-13} \mathrm{~s}$, which is 10 times longer than for the white noise. Furthermore, the pulse keeps its form during the scattering process.

The correlation between $\phi(r=0, t)$ and $\phi(r, t)$ is shown in Fig. 5, where the mean value is taken over all directions. Only one seed of the noise is used, which accounts for the observed fluctuations. The destruction of coherence is clearly seen. Defining the coherence time $t_{\text {coh }}$ as the time where $|\phi(r=0, t)|^{2}$ has decreased by a factor of $e$, the two approximations for white noise and time independent noise, respectively, result in

$$
\begin{aligned}
& t_{\text {coh }}(T=300 \mathrm{~K})=4 \times 10^{-14} \mathrm{~s}, \\
& t_{\text {coh }}(T=300 \mathrm{~K})=4 \times 10^{-13} \mathrm{~s},
\end{aligned}
$$


found from the numerical simulations.

The experiments by Möbius and Kuhn have shown that the exciton stays coherent during its lifetime $t_{\text {life }}$ [13]. Thus in order for the nonlinear model to be relevant the predicted coherence time must be larger than the lifetime found experimentally [13]

$$
t_{\text {life }}=10^{-10} \mathrm{~s}\left(\frac{T}{300 \mathrm{~K}}\right) .
$$

In Fig. 6 the numerically found coherence time for time independent noise and the lifetime, given by Eq. (46), are plotted versus temperature. The average has been taken over three seeds of the noise. The temperature dependence is seen to follow a power law

$$
t_{\mathrm{coh}}=4 \times 10^{-13} \mathrm{~s}\left[\frac{300 \mathrm{~K}}{T}\right]^{0.24} \text {. }
$$

The temperature where the curves for $t_{\text {coh }}$ and $t_{\text {life }}$ intersect is denoted $T_{0}$. At higher temperatures than $T_{0}$ the predicted coherence time is smaller than the lifetime found experimentally. Thus the nonlinear model is not relevant in this temperature regime. At temperatures lower than $T_{0}$ the coherence time is larger than the experimentally found lifetime. Thus the nonlinear model is relevant in this temperature regime, where it may describe the dynamics of the coherent exciton. With the parameters given in Table II $T_{0}=3 \mathrm{~K}$ is found from the numerical simulations.

\section{APPLICABILITY AND MODEL PARAMETERS}

Due to the very low critical temperature $T_{0}$, the applicability of the model seems limited. However, $T_{0}$ depends on the model parameters. We note that $t_{\text {coh }}$, as function of $D_{\text {disorder }}$, is given by

$$
t_{\mathrm{coh}}=\alpha\left(\frac{\hbar}{J_{0}}\right)\left(\frac{J_{0}^{2}}{l^{2} D_{\text {disorder }}}\right)^{\beta},
$$

where $\alpha$ and $\beta$ are found numerically to be

$$
\alpha=8.07, \beta=0.24 \text {. }
$$

Inserting the parameter values given in Table II yields Eq. (48). The average value of the power spectrum of the noise is not a constant, and Eq. (38) must be replaced by

\section{Initial condition}

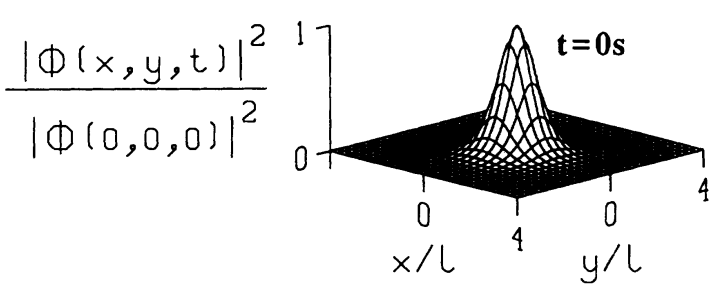

White noise
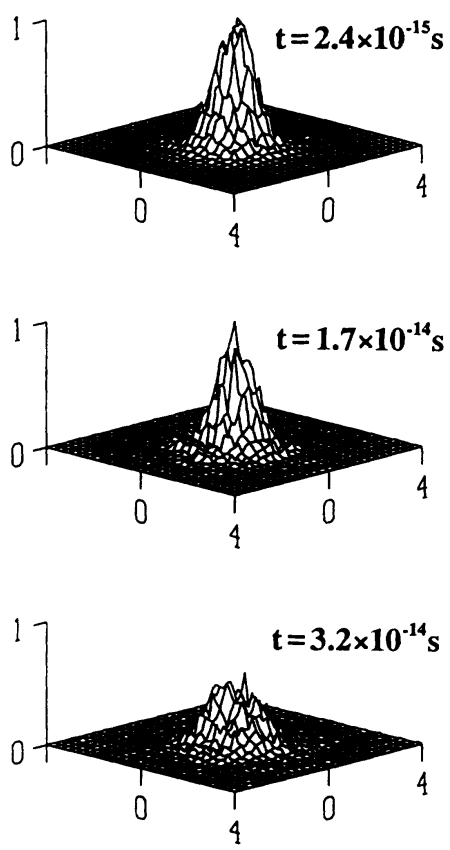

Disorder
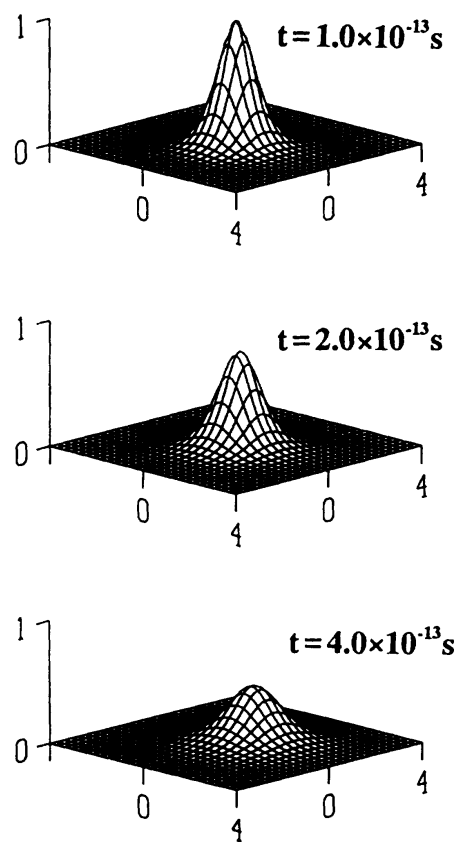

FIG. 4. Snapshots of the probability amplitude $|\phi(x, y, t)|^{2}$ for the numerical solution to Eq. (26). Initial condition is the GS solution, Eqs. (28) and (29). Parameters are taken from Table II. $T=300 \mathrm{~K}$. 

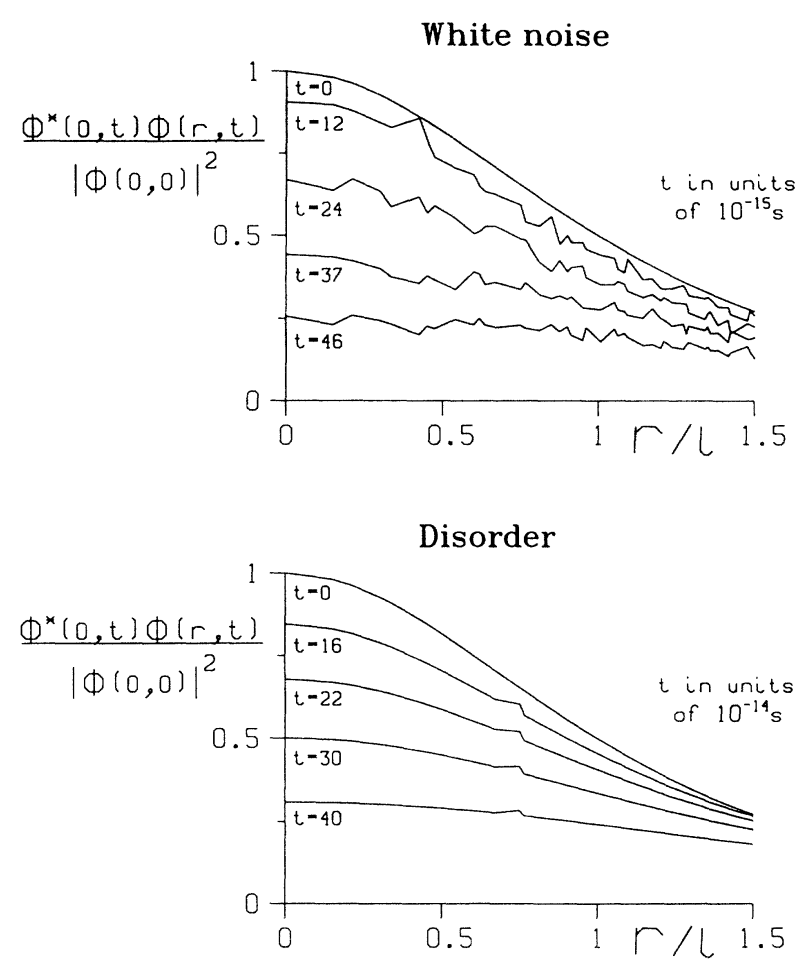

FIG. 5. $\phi^{*}(0, t) \phi(r, t)$ for the numerical solution to Eq. (26). Initial condition is the GS solution, given by Eqs. (28) and (29). Parameters are taken from Table II. $T=300 \mathrm{~K}$.

$$
D_{\text {disorder }}=\left\{\frac{2 \omega_{1} \chi^{2} \lambda k T}{\pi M \omega_{0}^{4} l^{2}}\right) A(\epsilon),
$$

where the average $A(\epsilon)$ can be written as a function of $\epsilon=\lambda /\left(2 \omega_{0}\right)$ only

$$
\begin{aligned}
A(\epsilon)=\int_{0}^{1}\left[\frac{\sin (\pi x)}{\pi x}\right]^{2} \\
\qquad\left[\frac{1}{\left[1-\left(1-\epsilon^{2}\right) x^{2}\right]^{2}+4 \epsilon^{2}\left(1-\epsilon^{2}\right) x^{2}}\right] d x
\end{aligned}
$$

For the parameter values given in Table II, $A \approx \frac{2}{3}$ and Eq. (51) reduces to Eq. (38).

The dependence of the critical temperature on the model parameters can now be found by combination of Eqs. (47) and (49)

$$
\frac{T_{0}}{300 \mathrm{~K}}=g(\epsilon)\left(\frac{3.6 \times 10^{-21} \mathrm{~J}}{J_{0}}\right)^{0.61}
$$

where the function $g(\epsilon)$ is given by

$$
g(\epsilon)=4.4 \times 10^{-3} \times\left(\epsilon \sqrt{1-\epsilon^{2}} A\right)^{-0.19} .
$$

Here the normalization condition, given by Eq. (31), has been used to obtain Eq. (53). In Fig. 7 the resulting dependence of $T_{0}$ on $\epsilon$ is shown for different orders of magnitude of $J_{0}$. It is seen that decreasing $\epsilon$ by a factor

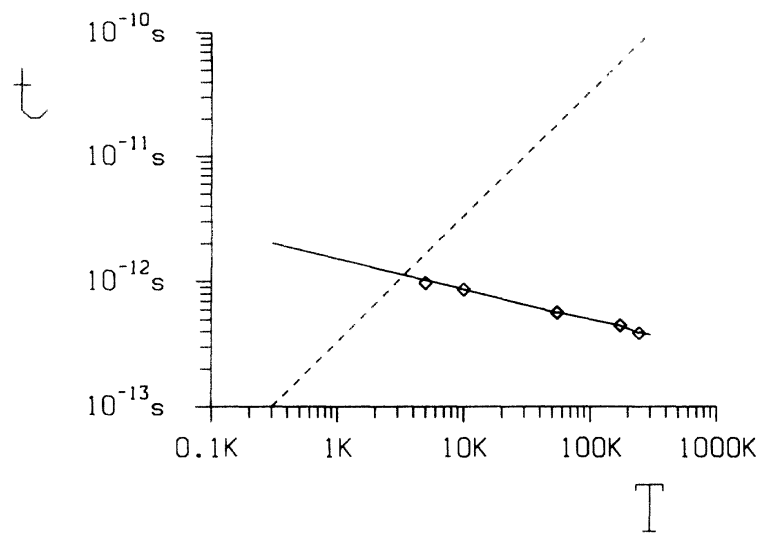

FIG. 6. Coherence time (solid curve) found by numerical solution of Eq. (26) with the GS solution, Eqs. (28) and (29) as initial condition, and the lifetime (dashed curve) given by Eq. (46), versus temperature. Parameters are taken form Table II.

of 100 only increases $T_{0}$ by $3.5 \mathrm{~K}$, where as a decrease in the dispersive coupling $J_{0}$ has a much strong effect.

A critical temperature around room temperature can be obtained for $J_{0} \propto 10^{-24} \mathrm{~J}$ and $\lambda \propto 10^{9} \mathrm{~s}^{-1}$, e.g. [to fulfill the normalization condition, Eq. (31), $\chi$ must then be decreased to be $\propto 10^{-11} \mathrm{~N}$ ]. In order that the proposed nonlinear continuum model be relevant at room temperature, the dispersive dipole-dipole coupling, as well as the nonlinear exciton-phonon coupling, must thus be weaker.

\section{CONCLUSION}

A nonlinear model of monolayer Scheibe aggregates has been derived, describing the dynamics of coherent excitons. Thermal fluctuations of the molecules have been introduced through additive Gaussian white noise in the phonon system, corresponding to thermal generation and destruction of phonons. This leads to multiplicative noise in the exciton system, corresponding to destruction of coherence through scattering by the fluctuating phonons.

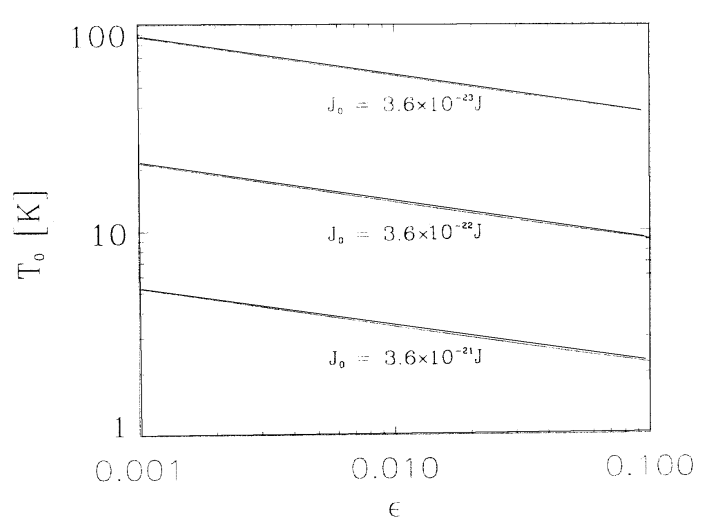

FIG. 7. Critical temperature $T_{0}$ versus $\epsilon$, as given by Eq. (53) for dipole-dipole coupling $J_{0}=3.6 \times 10^{-21}, 3.6 \times 10^{-22}$, and $3.6 \times 10^{-23} \mathrm{~J}$. 
The equation governing the dynamics of the excitons is the DST equation with noise, which is approximated by the two dimensional NLS equation with noise. Thus the domain of coherence observed in several experiments is modeled as the GS solution to this equation. The parameters in the model have been estimated for the oxacyanine Scheibe aggregate studied experimentally by Möbius and Kuhn [13]. However, only order of magnitude estimates could be given for essential parameters such as the phonon eigenfrequency, the linewidth of the infrared absorption spectrum, and the exciton-phonon coupling parameter, due to lack of experimental data.

The spectrum of the resulting noise is complicated and it is not practically possible to simulate numerically with the large number of molecules, which is desirable. Instead two approximations have been considered: pure white noise and time independent, spatially white noise. These are expected to give the limiting values of the coherence time.

The white noise is a poor approximation, since the energy content is infinite, which contrasts the finite energy of the real noise. Correspondingly a very short coherence time is found at room temperature, $t_{\mathrm{coh}}=4 \times 10^{-14} \mathrm{~s}$.

The time independent noise, which simply corresponds to disorder in the arrangement of the molecules, has approximately the same energy content as the real noise. At room temperature this approximation gives the coherence time $t_{\text {coh }}=4 \times 10^{-13} \mathrm{~s}$, the temperature dependence again being given by a power law $t_{\mathrm{coh}} \propto T^{-0.24}$.

Experiments show that the exciton stay coherent during its lifetime, which is proportional to the temperature, and $10^{-10} \mathrm{~s}$ at room temperature [13]. From this fact it is found that the proposed nonlinear model is relevant only at temperatures below $3 \mathrm{~K}$. In this temperature range the predicted coherence time is larger than the experimentally found lifetime.
The dependence of the limiting temperature on the model parameters has been found, showing that the dispersive dipole-dipole coupling and the exciton-phonon coupling must be decreased significantly in order for the model to be relevant at room temperature.

Using the white noise approximation instead of the disorder approximation, the power $\beta$ in Eq. (49) increases [Eq. (46) and (40)]. As a result the critical temperature will decrease. The correct colored noise will give a critical temperature between these two limits.

In the 2D continuum approximation the ground state solution is the only stable excitation [24], implying the normalization condition, Eq. (31). In the discrete version of the model, Eq. (22), this unphysical bond between the dipole-dipole coupling and the nonlinearity parameter is not present. Thus the exciton-phonon coupling can be reduced without changing the dipole-dipole coupling, in agreement with the results obtained in Refs. [18] and [19].

The conclusion that nonlinearity and solitary waves are only relevant below a certain temperature is well known from the Davydov model of energy transport in protein [25]. Here solitons are found to exist at $T=0 \mathrm{~K}$, but agreement has not yet been reached on whether they exist for sufficiently long times at physiological temperatures $[37,43]$.

\section{ACKNOWLEDGMENTS}

We wish to thank $\mathrm{H}$. Kuhn for providing experimental data for the oxacyanine dye LB film and A. C. Scott for many helpful discussions and valuable suggestions. O.B. acknowledges The Danish Technical Research Council for financial support under Grant No. 16-5009-1 PG. Y.B.G. acknowledges The Technical University of Denmark for additional support.
[1] A. S. Davydov, Theory of Molecular Excitons (Plenum, New York, 1971).

[2] R. S. Knox, in Primary Processes of Photosynthesis, edited by J. Barber (North-Holland, Amsterdam, 1977), p. 55.

[3] I. Langmuir, J. Am. Chem. Soc. 39, 1848 (1917).

[4] K. B. Blodgett, J. Am. Chem. Soc. 57, 1007 (1935).

[5] R. H. Tredgold, Rep. Prog. Phys. 50, 1609 (1987).

[6] L. M. Blinov, Russ. Chem. Rev. 52, 713 (1983).

[7] H. Bücher and H. Kuhn, Chem. Phys. Lett. 6, 183 (1970).

[8] G. Scheibe, Angew. Chem. 49, 563 (1936).

[9] G. Feher and M. Y. Okamura, in The Photosynthetic Bacteria, edited by R. K. Clayton and W. F. Sirtronm (Plenum, New York, 1978), p. 349.

[10] P. B. Gilman, Phot. Sci. Eng. 18, 418 (1974).

[11] Y. Wang, Chem. Phys. Lett. 126, 209 (1986).

[12] D. Möbius and H. Kuhn, Isr. J. Chem. 18, 375 (1979).

[13] D. Möbius and H. Kuhn, J. App. Phys. 64, 5138 (1988).

[14] S. De Boer and D. A. Wiersma, Chem. Phys. Lett. 165, 45 (1990).

[15] T. Itoh, T. Ikehara, and Y. Iwabuchi, J. Lumin. 45, 29 (1990).

[16] J. Feldman, G. Peter, E. O. Göbel, P. Dawson, K. Moore, C. Foxon, and R. J. Elliott, Phys. Rev. Lett. 59, 2337
(1987).

[17] P. O. J. Scherer and S. F. Fischer, in Time-Resolved Vibrational Spectroscopy, edited by A. Laubereau and M. Stockburger (Springer, New York, 1985), p. 297.

[18] J. Grad, G. Hernandez, and S. Mukamel, Phys. Rev. A 37, 3835 (1988); F. C. Spano, J. R. Kuklinski, and S. Mukamel, Phys. Rev. Lett. 65, 211 (1990).

[19] E. A. Bartnik and J. A. Tuszynski, Phys. Rev. E 48, 1516 (1993).

[20] G. C. Huth, F. Gutmann, and G. Vitiello, Phys. Lett. A 140, 339 (1989).

[21] V. E. Zakharov and A. B. Shabat, Zh. Eksp. Teor. Fiz. 61, 118 (1971) [Sov. Phys. JETP 34, 62 (1972)].

[22] P. S. Lomdahl, O. H. Olsen, and P. L. Christiansen, Phys. Lett. 78A, 125 (1980).

[23] P. L. Christiansen, S. Pagano, and G. Vitiello, Phys. Lett. A 154, 381 (1991); P. L. Christiansen, O. Bang, S. Pagano, and G. Vitiello, Nanobiology 1, 229 (1992).

[24] J. J. Rasmussen and K. Rypdal, Phys. Scr. 33, 481 (1986).

[25] A. S. Davydov, J. Theor. Biol. 38, 559 (1973).

[26] N. W. Ashcroft and N. D. Mermin, Solid State Physics (Saunders College Publishing, Orlando, 1976), Chap. 22-23. 
[27] A. S. Davydov, Quantum Mechanics, 2nd ed. (Pergamon, Oxford, 1965), Chap. 20.

[28] R. Kubo, Rep. Prog. Phys. 29, 255 (1966).

[29] P. S. Lomdahl and W. C. Kerr, Phys. Rev. Lett. 55, 1235 (1985).

[30] J. C. Eilbeck, P. S. Lomdahl, and A. C. Scott, Physica D 16, 318 (1985).

[31] M. Desaix, D. Anderson, and M. Lisak, J. Opt. Soc. Am. 8, 2082 (1991).

[32] D. Anderson, M. Bonnedal, and M. Lisak, Phys. Fluids 22, 1838 (1979).

[33] K. Rypdal and J. J. Rasmussen, Phys. Scr. 33, 498 (1986).

[34] K. Rypdal, J. J. Rasmussen, and K. Thomsen, Physica D 16, 339 (1985).

[35] O. Bang, J. J. Rasmussen, and P. L. Christiansen, Nonlinearity 7, 205 (1994).
[36] V. Czikkely, H. D. Försterling, and H. Kuhn, Chem. Phys. Lett. 6, 297 (1970).

[37] A. C. Scott, Phys. Rep. 217, 1 (1992).

[38] F. If, M. P. Soerensen, and P. L. Christiansen, Phys. Rev. B 32, 1512 (1985).

[39] N. G. Van Kampen, Phys. Rep. 24, 171 (1976).

[40] O. Bang, P. L. Christiansen, Y. B. Gaididei, F. If, and K. $\emptyset$. Rasmussen (unpublished).

[41] O. Bang, Ph.D. thesis, Technical University of Denmark, DK-2800 Lyngby, Denmark, 1993.

[42] R. T. Taha and M. J. Ablowitz, J. Comput. Phys. 55, 203 (1984).

[43] Davydov's Soliton Revisited: Self-Trapping of Vibrational Energy in Protein, Vol. 243 of NATO Advanced Study Institute, Series B: Physics, edited by P. L. Christiansen and A. C. Scott (Plenum, New York, 1990). 